Plast Surg 1998;51:431-5.

2. Hallock GG. The medial sural(MEDIAL

GASTROCNEMIUS) perforator local flap. Ann Plast

Surg 2004;53:501-5.

3. Innocenti M, Menichini G, Baldrighi C, et al. Are there risk factors for complications of perforator-based propeller flaps for lower-extremity reconstruction? Clin Orthop Relat Res 2014;472:2276-86.

4. Kosutic D, Pejkovic B, Anderhuber F, et al. Complete mapping of lateral and medial sural artery perforators: anatomical study with Duplex-Doppler ultrasound correlation. J Plast Reconstr Aesthet Surg 2012;65:15306.

5. Wong $\mathrm{CH}$, Tan BK. Intermittent short saphenous vein phlebotomy: an effective technique of relieving venous congestion in the distally based sural artery flap. Ann Plast Surg 2007;58:303-7.

\section{The Use of a Mechanical Stapler in Jejunal Free Flaps in Laryngopharyngectomy Defects}

Jaebeom Park ${ }^{1}$, Nakheon $\mathrm{Kang}^{1}$, Sang $\mathrm{Ha} \mathrm{Oh}^{1}$, Sang Il Lee ${ }^{2}$, Seung Han Song ${ }^{1}$

Departments of ${ }^{1}$ Plastic and Reconstructive Surgery and ${ }^{2}$ Surgery, Chungnam National University School of Medicine, Daejeon, Korea

\section{Correspondence: Seung Han Song}

Department of Plastic and Reconstructive Surgery, Chungnam National University School of Medicine, 282 Munhwa-ro, Jung-gu, Daejeon 35015, Korea

Tel: +82-42-280-7381, Fax: +82-42-280-7384, E-mail: teddylisk@naver.com

This work was supported by the research funds of Chungnam National University.

No potential conflict of interest relevant to this article was reported.

Received: 19 May 2015 • Revised: 14 Jul 2015 • Accepted: 7 Sep 2015 pISSN: 2234-6163・ elSSN: 2234-6171

http://dx.doi.org/10.5999/aps.2015.42.6.815 • Arch Plast Surg 2015;42:815-818

Copyright (C) 2015 The Korean Society of Plastic and Reconstructive Surgeons This is an Open Access article distributed under the terms of the Creative Commons Attribution Non-Commercial License (http://creativecommons.org/licenses/by-nc/3.0/ which permits unrestricted non-commercial use, distribution, and reproduction in any medium, provided the original work is properly cited.

The reconstruction of laryngopharyngectomy defects requires the creation of a tubular passage that connects the pharyngeal remnant to the cervical esophagus. Tubed fasciocutaneous flaps (e.g., from the anterolateral thigh or radial forearm) are most commonly used, because they permit good postoperative speech and result in low systemic morbidity. However, if a fasciocutaneous flap is unavailable due to a lack of perforators or an obese thigh, the next choice is a jejunal flap. The most common method of securing visceral graft anastomoses was previously one or two layers of hand sewing. However, circular mechanical staplers have been commonly used by general surgeons for enteric anastomosis and lead to similar or lower anastomotic leakage and stricture rates than hand sewing $[1,2]$. Stapled anastomoses may also reduce the mean operating time by reducing the time required for anastomosis, and also probably reduce blood loss and the likelihood of intraoperative contamination [3]. The ischemic time seems to be a very important factor affecting the likelihood of postoperative complications, especially for free flaps. In particular, the intestine is more vulnerable to ischemia than the skin and bone [4]. In this report, the use of circular mechanical stapling for jejunoesophageal anastomosis was reviewed to assess whether it is a good alternative to hand-sewn anastomoses in the reconstruction area.

Jejunal free flap surgery was performed in six patients with a total laryngopharyngectomy defect between March 2009 and January 2013. Total laryngopharyngectomy was performed to treat hypopharyngeal cancer in four cases and to treat glottis cancer in two cases. All patient specimens were diagnosed as squamous cell carcinoma by a

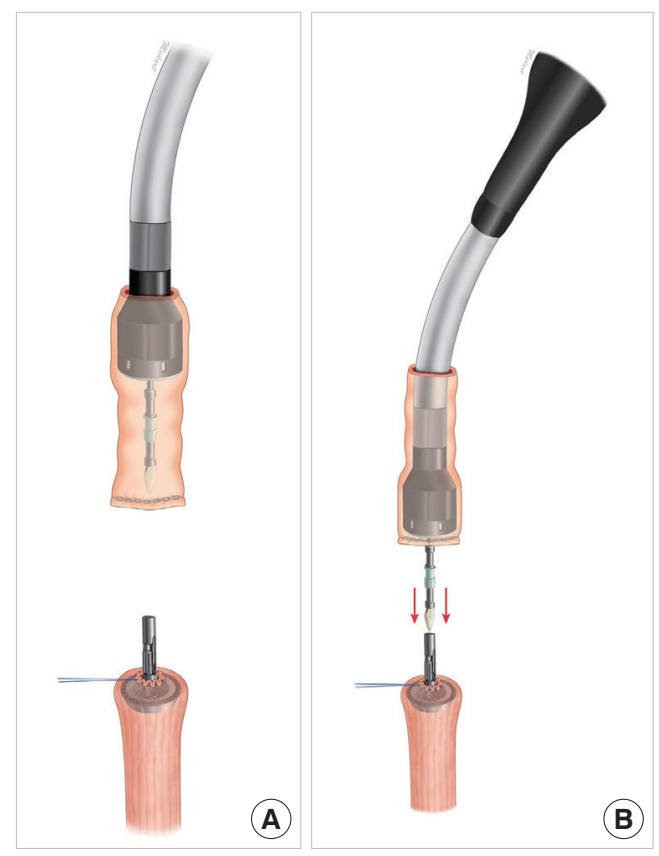

Fig. 1.

Diagram of the anastomosis process. (A) Diagram showing the proximal esophagus with the trocar in place and retraction (purse-string) sutures for dilating and sizing the esophageal lumen. (B) The pierced jejunum is seated within the stapler cartridge. 
Fig. 2.

Diagram of the stapling process. (A) Firing the stapler.

After firing, the end-to-end anastomosis device is removed gently via the jejunal flap lumen. (B) Inset, jejunum flap.
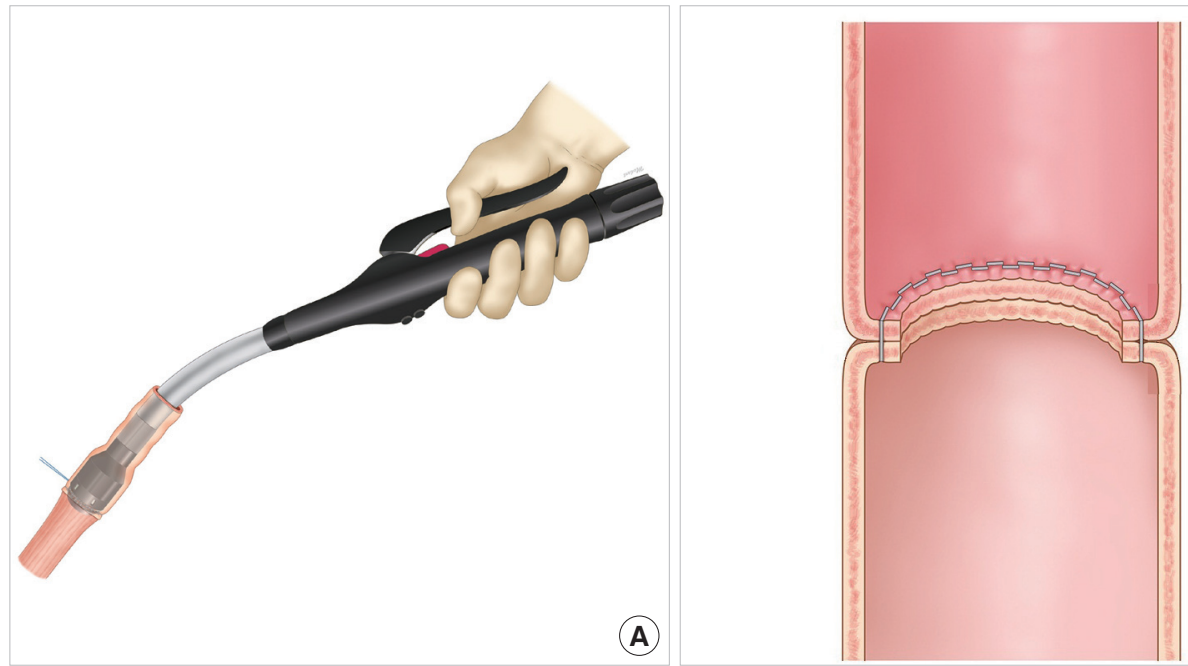

(B)

pathologist. A single general surgeon harvested the jejunal flap segment in all six cases. A distal jejunoesophageal anastomosis was performed using a 25-mm end-to-end anastomosis (EEA) circular mechanical stapler (Covidien, Mansfield, MA, USA) in all cases.

The anastomosis process for the flap was as follows. First, traction sutures were placed in the cervical esophagus to dilate the esophageal lumen. Purse-string sutures were placed at the end of the esophagus prior to stapling. The jejunal flap was pierced with a sharp trocar end, seated in position within the stapler cartridge, and the safety lock was then removed (Fig. 1). Stapling was performed by triggering. After confirming anastomosis of the entire tubular margin, the device was gently removed via the flap lumen (Fig. 2). Proximal flap anastomosis was performed using hand-sewn sutures in an interrupted fashion. At this point, the conduit was trimmed to eliminate redundancy. The suturing was performed with a double layer of interrupted (Covidien, Mansfield, MA, USA) (Fig. 3). We then performed revascularization of the pedicle vessels. We recorded the duration of stapler usage in each case. Two to three months after surgery, patients were assessed for anastomotic leakage or stricture by barium esophagography and endoscopy.

No patient experienced a fistula from the enteroanastomosis site. One of the six patients (16.7\%) showed mild tract stenosis at the jejunoesophageal anastomosis at a three-month follow-up examination. No cases of flap necrosis were observed after surgery. Five patients achieved a satisfactory postoperative outcome. No case of donor site infection, abdominal wound dehiscence, or bowel obstruction was noted. The average follow-up period was 18 months. The entire process of stapling took three to five minutes in all cases. The average procedure time for EEA stapling was 265.7 seconds (range, 191-340 seconds).

Reconstructing circumferential laryngopharyngeal and esophageal defects remains a technical challenge, and surgeons require an efficient and effective method of reducing operative time and perioperative stress. Bowel anastomoses were conventionally performed using hand-sewn techniques in the past. Since its introduction in 1960s, the advantages of the mechanical stapler reported in the literature can be summarized as follows: saving time, creating a regular uniform anastomosis, and involving less operator dependency with the corresponding relief of surgical stress. Due to these advantages, mechanical staplers have been widely used in various resection operations in the specialties of general surgery and thoracic surgery. In particular, during free flap procedures, the mean operative time is critical to the success of surgery, as prolonged operative times increase the intraoperative infection risk and blood loss and can induce perioperative complications and flap injury [3]. Nevertheless, few studies have described the use of mechanical staplers in the field of plastic and reconstructive surgery, especially in laryngopharyngeal defect reconstruction [2]. The critical ischemic time varies among different flap types. Fasciocutaneous flaps seem to be more resistant to ischemia than flaps from some other tissues, such as muscle and intestinal mucosa. However, the jejunum is one of the most vulnerable tissues to ischemic injury. Prolonged ischemic time can result in bowel stenosis [4]. This susceptibility of 

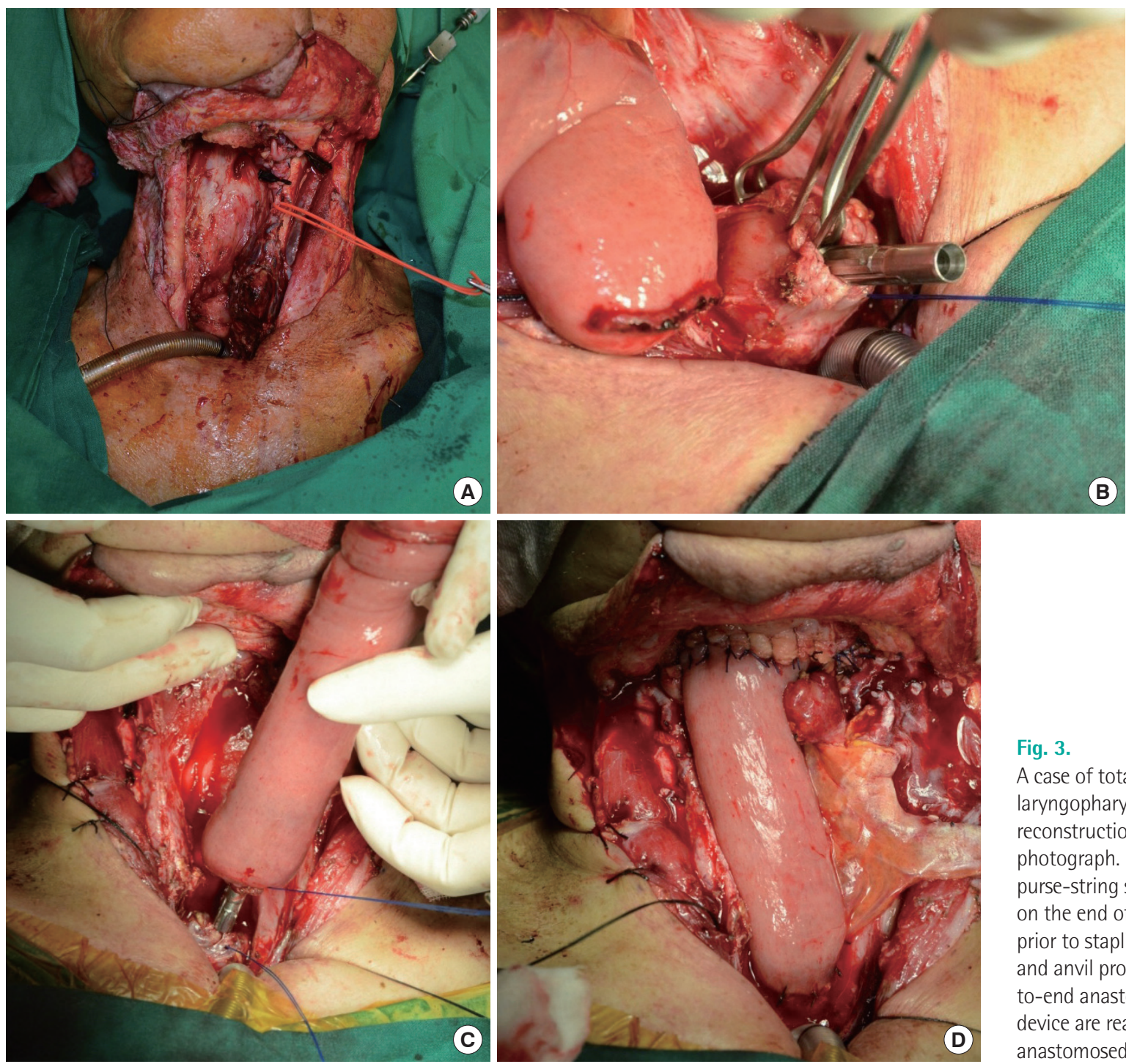

Fig. 3.

A case of total

laryngopharyngeal defect reconstruction. (A) Preoperative photograph. (B) The trocar and purse-string sutures are placed on the end of the esophagus prior to stapling. (C) The trocar and anvil probe of the endto-end anastomosis stapler device are reassembled. (D) The anastomosed jejunum flap.

jejunum flaps makes places stress on surgeons during the anastomosis process. The present study shows that the stapling procedure can be completed in three to five minutes. Moreover, in a previous study on colorectal surgery, the time to complete two layers of hand sewing in an anastomosis was found to be 10-20 minutes longer than stapled anastomosis [1]. Enteroanastomosis is not a common procedure in plastic surgery. EEA staplers lead to similar outcomes regardless of the skill level of the surgeon and are less operator-dependent [3]. Due to our limitation of a small sample size, we were not able to conduct a comparative and randomized study with a control group of hand-sewn anastomoses. We therefore reviewed the published literature. The presence of clinically significant differences in the formation of strictures and fistulae between stapled jejunoesophageal anastomoses and hand-sewn anastomoses remain controversial [5]. However, many authors have concluded that both methods are safe, and in some studies, no significant differences in fistula or stricture formation were observed between stapled and hand-sewn anastomoses [2,3]. In order to minimize the risk of fistula formation, we carefully examined tissue specimens excised using the stapler and inspected the stapled line for hemostasis after flap anastomosis. One patient developed strictures after three months, and the symptoms improved after balloon dilation of the midconduit stricture and a few months of a liquid diet. All six of our patients underwent esophageal reconstructive surgery with an EEA stapler and achieved good results without fistula formation or flap necrosis.

In conclusion, EEA stapling has the following advantages compared to using hand-sewn sutures: a shorter operative time, similar clinical results, and less 
operator dependence. We propose this convenient method for free jejunal grafts for laryngopharyngeal defects.

\section{References}

1. Dziki AJ, Duncan MD, Harmon JW, et al. Advantages of handsewn over stapled bowel anastomosis. Dis Colon Rectum 1991;34:442-8.

2. Schneider DS, Gross ND, Sheppard BC, et al. Reconstruction of the jejunoesophageal anastomosis with a circular mechanical stapler in total laryngopharyngectomy defects. Head Neck
2012;34:721-6.

3. Law S, Fok M, Chu KM, et al. Comparison of handsewn and stapled esophagogastric anastomosis after esophageal resection for cancer: a prospective randomized controlled trial. Ann Surg 1997;226:16973.

4. Siemionow M, Arslan E. Ischemia/reperfusion injury: a review in relation to free tissue transfers. Microsurgery 2004;24:468-75.

5. Fok M, Ah-Chong AK, Cheng SW, et al. Comparison of a single layer continuous hand-sewn method and circular stapling in 580 oesophageal anastomoses. $\mathrm{Br} \mathrm{J}$ Surg 1991;78:342-5. 